\title{
Mindfulness y el Abordaje del TDAH en el Contexto Educativo ${ }^{1}$ MindFulNESS E A ABORDAGEM DO TDAH NO CONTEXTO EDUCACIONAL Mindfulness and the APPROACH OF ADHD in the EDUCATIONAL CONTEXT
}

\author{
Irene López SECANELL² \\ Sabina Pastor NÚNEZ ${ }^{3}$
}

\begin{abstract}
RESUMEN: El objetivo del presente trabajo ha sido la realización de una revisión sistemática de estudios sobre la eficacia de las intervenciones basadas en mindfulness en el tratamiento del TDAH para su posible puesta en práctica en el contexto educativo. Así mismo también se ha analizado la metodología y el diseño de cada estudio. El proceso de investigación se realizó en las bases de datos PsycINFO, ERIC, Dialnet plus, Roderic y Google Scholar, utilizando palabras clave y determinados criterios de inclusión y de exclusión. Solo seis artículos de 121 cumplían estos criterios. Los resultados muestran como las investigaciones evidencien una reducción de la sintomatología principal del TDAH mediante el mindfulness. Al mismo tiempo, concluimos la falta de estudios y programas de mindfulness y TDAH inclusivos en ámbitos educativos.
\end{abstract}

PALABRAS CLAVE: Mindfulness. TDAH. Educación. Intervención.

RESUMO: O objetivo do presente trabalho foi a realização de uma revisâo sistemática de estudos sobre a eficácia das intervençôes baseadas em mindfulness no tratamento do TDAH para sua possível implementação no contexto educacional. Da mesma forma, também foram analisados a metodologia e o procedimento de cada estudo. O processo de pesquisa foi realizado nas bases de dados PsycINFO, ERIC, Dialnet plus, Roderic e Google Scholar, utilizando palavras-chave e determinados critérios de inclusão e exclusão. Apenas seis artigos dentre 121 preencheram esses critérios. Os resultados mostram como as pesquisas evidenciam uma redução da sintomatologia principal do TDAH através do mindfulness. Ao mesmo tempo, concluímos a falta de estudos e programas de mindfulness e TDAH inclusivos em contextos educacionais.

PALAVRAS-CHAVE: Mindfulness. ADHD. Educação. Intervenção.

ABSTRACT: The aim of this work has been to carry out a systematic review of studies on the effectiveness of interventions based on mindfulness in the treatment of ADHD for its possible implementation in the educational context. Likewise, the methodology and design of each study has also been analyzed. The research process was realized in PsycINFO, ERIC, Dialnet plus, Roderic and Google Scholar databases, using keywords and certain inclusion and exclusion criteria. Only six articles out of 121 met these criteria. The results show how research evidences a reduction of the main symptomatology of ADHD through mindfulness. At the same time, we concluded the lack of studies and inclusive programs of mindfulness and ADHD in educational settings.

Keywords: Mindfulness. ADHD. Education. Intervention.

\section{INTRODUCCIÓN}

En los últimos años, las investigaciones centradas en el trastorno por déficit de atención (TDAH) se han incrementado, ya que es uno de los trastornos más frecuentes en niños/as y adolescentes (Catalá-López y Hutton, 2018). La inatención, la hiperactividad y la impulsividad se presentan como las principales características de este trastorno junto a la baja tolerancia a la frustración, la irritabilidad y la variabilidad del estado de ánimo. Estos síntomas interfieren el

\footnotetext{
${ }^{1}$ http://dx.doi.org/10.1590/S1413-65382519000100011

${ }^{2}$ Florida Universitaria, Catarroja, València, España. irlopez@florida-uni.es.

ORCID https://orcid.org/0000-0002-7720-1658

${ }^{3}$ Florida Universitaria, Catarroja, València, España. sabinapastor1@hotmail.com.

ORCID https://orcid.org/0000-0001-8124-5146
} 
ámbito social y laboral, pero sobretodo hace que se evidencien problemas de ajuste en el ámbito escolar (Sánchez y González, 2013).

Existen numerosos estudios sobre las diferentes intervenciones y tratamientos del TDAH. Los que cuentan actualmente con una mayor evidencia empírica son los estimulantes de sistema nervioso central, la modificación de la conducta y la combinación de los dos (Miranda, Jarque, y Tárraga, 2006). En estos estudios se evidencia como los procedimientos más habituales de atención psicopedagógica en la escuela son: la utilización de técnicas de modificación de la conducta, técnicas cognitivo-conductuales, la formación de padres y la remisión de los niños a especialistas en medicina. Aunque estas técnicas y estrategias de intervención resultan una herramienta eficaz para la mejora de la adaptación escolar de los nińos con TDAH (Miranda et al., 2006) son diferentes factores los que pueden influir en la evolución y pronóstico de los niños y nińas con TDAH. Algunos de ellos son el nivel de gravedad inicial del trastorno comorbilidad con otros trastornos, el diagnóstico precoz, el perfil cognitivo-conductual y emocional de cada niño o niña, el ámbito socio-familiar, la influencia genética, dificultades de aprendizaje asociadas y el grado de éxito de las intervenciones llevadas a cabo (Orjales, 2000). Esta concepción implica una visión e intervención integral del niño o niña con TDAH que tenga en cuenta estos factores.

Generalmente, los tratamientos farmacológicos y conductuales son los más efectivos para este trastorno (Schachter, King, Langford, y Moher, 2001). No obstante, en los últimos años se ha incrementado el interés por tratamientos alternativos no farmacológicos a causa de la falta de efectividad de algunos tratamientos y sus efectos secundarios (Schachter et al., 2001). Frente a este escenario, dentro del ámbito de las intervenciones psicosociales, los programas basados en la práctica del mindfulness se rebelan como un tratamiento alternativo psicológico beneficioso. Según García, Demarzo y Modrego (2017) las intervenciones basadas en mindfulness han mostrado su eficacia contribuyendo de manera complementaria a la obtención de beneficios a nivel físico, emocional y cognitivo de los estudiantes, respecto a la conciencia de su cuerpo, la gestión y autocontrol de las emociones y la mejora de la concentración y estimulación de la creatividad. Como vemos, los beneficios físicos y psíquicos que aporta el mindfulness dan respuesta a la sintomatología propia del TDAH: inatención, hiperactividad e impulsividad. No obstante, aunque cada año se publica multitud de artículos que versan sobre la efectividad del mindfulness en el contexto educativo, pocos son los trabajos que muestran investigaciones sobre mindfulness realizadas en contextos educativos con casos de TDAH.

Por ello, el presente trabajo tiene la finalidad de realizar una revisión bibliográfica sobre la efectividad que tienen las intervenciones de mindfulness en el tratamiento del TDAH, con el fin de abordar la posibilidad de llevarlas a la práctica en el contexto educativo de manera inclusiva. Con ello pretendemos asentar unas bases teóricas que nos sirvan para conocer qué se ha investigado entorno al mindfulness y el TDAH para así tener los principios que nos deriven al planteamiento de futuras intervenciones didácticas en ámbitos escolares.

\subsection{Aproximación al CONCEPTo de TDAH deSDe El Ámbito ESCOLAR}

Según la American Psychiatric Association [APA] (2014), el TDAH es un trastorno del neuro-desarrollo cuya característica principal es un patrón persistente de inatención y/o hi- 
peractividad e impulsividad que interfiere en el funcionamiento o el desarrollo del niño (5\%) o nińa $(2,5 \%)$. La inatención o problemas que presentan los alumnos con TDAH para mantener la atención junto a la falta de persistencia y desorganización pueden comprometer la adquisición de las habilidades académicas, ya que en términos generales será función de la atención la facilitación del aprendizaje mediante la promoción de una mayor concentración y seguimiento activo de las tareas y explicaciones (Razza, Martin, y Brooks-Gunn, 2012).

Las últimas investigaciones llevadas a cabo por Barkley y Murphy (2010) hacen hincapié en que los niños/as con TDAH suelen presentar limitaciones en dos componentes de control: la impulsividad emocional y las dificultades en el autocontrol emocional. Estos déficits en auto-regulación pueden estar en la base de los problemas en las relaciones sociales de los niños/as con TDAH y definir un perfil temperamental caracterizado por una alta reactividad emocional y pobres habilidades de auto-regulación (Sánchez y González, 2013).

La confluencia de déficits cognitivos, falta de habilidades de auto-regulación y un perfil de temperamento basado en una alta reactividad emocional y baja auto-regulación, potencian el riesgo de que el alumnado con TDAH desarrolle una diversidad de problemas de ajuste en la escuela (Sánchez y González, 2013). Por ello, se hace necesaria la intervención en el contexto escolar mediante programas que tienen en cuenta la adaptación por parte del profesorado a las necesidades educativas específicas de todo su alumnado, incluidos los alumnos con TDAH, así como el entrenamiento en habilidades cognitivo-emocionales para darles la oportunidad de aumentar sus competencias. Como se ha mencionado en la introducción del presente articulo, se está incrementando el interés por tratamientos alternativos no farmacológicos como el mindfulness (Schachter et al., 2001; García et al., 2017). A continuación, vamos a hacer una aproximación al concepto de mindfulness en el contexto educativo y como éste se contempla como una posibilidad alternativa dentro de los tratamientos para el TDAH.

\subsection{Conceptualización Y BeNEFicios Del MindfulNesS}

El término mindfulness proviene de una traducción de la palabra sati, procedente de la lengua pali (García et al., 2017). Esta palabra hacía referencia a la conciencia, atención (conciencia focalizada) y recuerdo (entendido como la capacidad de recordarnos de ser conscientes). Su introducción en el mundo occidental fue realizada por Kabat-Zinn en 1978 a través del Programa de Mindfulnes Basado en la Reducción del Estrés (MBSR), con el que se quería ayudar a los pacientes del centro médico de la Universidad de Massachussets a reducir el estrés y el dolor crónico. Según Kabat-Zinn (2003, p. 145) podemos definir el mindfulness como "el estado particular de conciencia que surge al poner la atención, de forma intencional y sin juzgar, en el momento presente, viviendo la experiencia momento a momento”.

Kabat-Zinn (2003) propuso unos principios del mindfulness que se basaban en 9 características que se deben dar cuando practicamos esta técnica: no-enjuiciamiento, aceptación, confianza, paciencia, apertura de visión, simplicidad, desapego, generosidad y gratitud. En base a estos principios muchos los autores han planteado actividades para su desarrollo (Rechtscgaffen, 2016). No obstante, todos ellos se basan en ejercicios de meditación y respiración como medio para trabajarlos. Basándonos en Rechtscgaffen (2016) podemos clasificar las actividades de mindfulness en las siguientes categorías: 
- Incorporación: se basa en tomar consciencia de las sensaciones y las emociones que están viviendo en nuestro cuerpo y en el estado en que se encuentra nuestra mente cuando nos movemos o hacemos actividades como yoga, danza, Pilates, etc.

- Atención: se basa en ejercicios donde mediante la meditación se lleva la atención en las sensaciones de la respiración.

- Corazón pleno: se basa en ejercicios de imaginación donde se visualizan situaciones con otras personas y se toma consciencia de cómo estas nos afectan.

- Interconnexión: se basa en ejercicios de meditación que centran en la atención a los fenómenos exteriores (sonidos, olores, sabores, etc.) y cómo éstos nos afectan a nivel interno.

- Inteligencia emocional: propone ejercicios que facilitan ser testigos de nuestros juicios de valor, a reflejar nuestras proyecciones y trabajar con nuestras emociones a partir de la meditación.

- Comunicación mindful: se basa en deconstruir pautas de comunicación viejas y dañinas. Invita a observar nuestras intenciones subyacentes y los efectos de nuestros actos hacia los demás a partir de la meditación y la reflexión.

En los últimos años, las evidencias empíricas sobre los beneficios del mindfulness al contexto educativo se han ido incrementando y han ayudado a reafirmar los numerosos beneficios que implica esta práctica. Considerando la variedad de investigaciones y referentes teóricos que existen, a continuación, sintetizamos por categorías los distintos beneficios del mindfulness:

- $\quad$ Beneficios a nivel psicológico: reducción de los niveles de estrés (Anand y Sharma, 2014; Kuyken et al., 2013; Metz et al., 2013; Zenner, Herrleben-Kurz, y Walach, 2014), reducción de los niveles de ansiedad (Franco, Mañas, Cangas, y Gallego, 2011; Napoli, Krech, y Holley, 2005; Potek, 2012), reducción de los niveles de depresión (Britton, et al., 2014; Kuyken et al., 2013; Schonert-Reichl et al., 2015; Zoogman, Goldberg, Hoyt, y Miller, 2015), reducción de los niveles de afecto negativo y incremento de los niveles de afecto positivo (Britton et al., 2014; Broderick y Metz, 2009; Klingbeil et al., 2017; Schonert-Reichl y Lawlor, 2010), reducción de los niveles de cansancio, dolor y quejas psicosomáticas (Broderick y Metz, 2009; Metz et al., 2013).

- Beneficios relacionados con el aumento de sentimientos y constructos positivos: sensaciones de calma, relajación, sueño mejorado, menor reactividad, incremento de la auto-cuidado, auto-conciencia y un sentido de interconexión o interdependencia con la naturaleza (Wall, 2005), mejora de los niveles de optimismo (Schonert-Reichl y Lawlor, 2010; Schonert-Reichl et al., 2015), mejora del auto-concepto (Franco et al., 2011; Klingbeil et al., 2017).

- Beneficios en relación con la regulación emocional: mayor conciencia y claridad emocional (Broderick y Metz, 2009; Schonert-Reichl et al., 2015) y autocontrol de emociones negativas (Broderick y Metz, 2009; Rosaen y Benn, 2006; Schonert-Reichl et al., 2015).

- Beneficios en la función cognitiva: aumento en los niveles de atención (Napoli, et al., 2005; Saltzman y Goldin, 2008; Schonert-Reichl y Lawlor, 2010; Tang, Yang, Leve, 
y Harold, 2012), aumento en la autorregulación y función ejecutiva (Biegel y Brown, 2010; Desmond, Hanich, y Millersville, 2010; Flook et al., 2010; Meiklejohn et al., 2012; Tang et al., 2012; Zenner, et al., 2014), mejoras en otras variables relacionadas con el funcionamiento cognitivo, velocidad de procesamiento de información, inteligencia práctica, independencia de campo y creatividad (So y Orme-Johnson, 2001) e incremento de los niveles de creatividad verbal (Franco, 2009).

- Beneficios en el ámbito social y escolar: incremento de las habilidades sociales y de las conductas socialmente competentes: incremento de las HHSS (Biegel y Brown, 2010; Napoli, et al., 2005) incremento en las conductas socialmente competentes (Klingbeil et al., 2017; Meiklejohn et al., 2012; Schonert-Reichl y Lawlor, 2010; Schonert-Reichl et al. 2015; Waters, Barsky, Ridd, y Allen, 2015), mejora de la inteligencia social (Rosaen y Benn, 2006), mejora del comportamiento del alumnado y del clima del aula; reducción en comportamiento disruptivo (Joyce, Etty-Leal, Zazryn, y Hamilton, 2010), reducciones en comportamientos relacionados, con el TDAH (Klatt, Harpster, Browne, White, y Case-Smith, 2013), reducción de los niveles de agresión e incremento de los niveles de aceptación (Schonert-Reichl et al., 2015), mejora del auto-concepto académico y del rendimiento académico (Anand y Sharma, 2014; Felver, Celis-De Hoyos, Tezanos, y Singh, 2016; Franco et al., 2011; Klingbeil et al., 2017; León, 2008; Schonert-Reichl et al., 2015; Tang et al., 2012).

A la luz de estos resultados, las intervenciones basadas en la atención plena en las escuelas se evidencian como una alternativa para la mejora de la auto-regulación emocional y atencional del alumnado, favoreciendo disposiciones prosociales, su adaptación al medio y logro, por tanto, de un mayor bienestar psicológico.

\subsection{El MindfulNesS EN el CONTEXTo eduCATIVo}

Basándonos en los numerosos beneficios del mindfulness que se han mostrado en el apartado anterior podemos afirmar como la práctica del mindfulness, podría ayudar al alumnado a reducir el estrés y aumentar su propio bienestar, para alcanzar y optimizar el aprendizaje y mejorar sus relaciones interpersonales. Según Zenner et al. (2014) en el contexto educativo, el mindfulness o atención plena puede ser entendida como una base y una condición previa para la educación, ya que el mindfulness aporta numerosos beneficios que se traducen en una mejora de nuestra salud (Anand y Sharma, 2014; Britton et al., 2014; Broderick y Metz, 2009; Franco et al., 2011; Kuyken et al., 2013; Metz et al., 2013; Napoli, et al., 2005; Potek, 2012; Schonert-Reichl et al., 2015; Schonert-Reichl y Lawlor, 2010).

García et al. (2017) indican que la implementación de programas basados en la atención plena en las escuelas se presenta, para un número creciente de centros educativos, como una vía de ayuda para el alumnado respecto a su autorregulación emocional y atencional, favoreciendo su adaptación al contexto y para alcanzar un mayor bienestar psicológico. A nivel nacional, los programas de mindfulness que se han introducido en centros educativos son "Programa "aulas felices", Programa "TREVA", programa "Crecer Respirando" y programa "Escuelas Despiertas”. Así como existen numerosos programas de mindfulness en el contexto educativo, cuando buscamos programas de mindfulness que contemplen necesidades educati- 
vas especiales como el TDAH solo encontramos el programa "MyMind" (Bögels, Hoogstad, Van Dun, De Shutter, y Restifo, 2008). Este es un programa de intervención para padres e hijos con TDAH y fue desarrollado y validado en 2008 por Susan Bögels, con resultados muy efectivos en padres e hijos. Este programa ha sido aplicado en diferentes trastornos externalizantes entre los que se encuentra el TDAH, trastorno negativista desafiante, trastorno de conducta y Asperger.

La existencia de programas como "MyMind" nos abre una puerta a la intervención inclusiva del TDAH al contexto educativo a través de tratamientos alternativos como el mindfulness. No obstante, también nos evidencia la necesidad de realizar, aplicar y publicar más programas a nivel nacional que tengan una visión inclusiva de todo el alumnado.

\section{MÉTODO}

\subsection{ESTRATEGIA DE BÚSQUEDA BIBLIOGRÁFICA}

La búsqueda de documentación se realizó a lo largo de un período de siete meses (septiembre 2017-abril 2018). El análisis de la revisión bibliográfica se concretó en tres fases. La primera fase consistió en una primera revisión en las siguientes bases de datos para la búsqueda de estudios o trabajos científicos sobre la temática en cuestión: PsycINFO, ERIC, Dialnet plus, Roderic y Google Scholar. En estas bases las palabras de búsqueda utilizadas fueron las siguientes: "mindfulness", "intervention", "therapy", "treatment", "attention deficit and hyperactivity disorder (ADHD)", tanto en inglés como en español. Además, se utilizaron operadores booleans (Y / AND, O / OR, NO / NOT, etc). De esta manera se accedieron a las publicaciones existentes sobre terapias o tratamientos basados en mindfulness para el TDAH. En la "Tabla 1" se exponen el número total de artículos obtenidos en la primera fase de la revisión bibliográfica:

\begin{tabular}{lllc}
\multicolumn{1}{c}{ Bases de datos } & \multicolumn{1}{c}{ Palabras clave } & Booleans & No artículos \\
\hline Dialnet plus & $\begin{array}{l}\text { Mindfulness, TDAH, ADHD, terapia, therapy, } \\
\text { tratamiento, treatment }\end{array}$ & AND, OR & 8 \\
ERIC & $\begin{array}{l}\text { Mindfulness, TDAH, ADHD, terapia, therapy, } \\
\text { tratamiento, treatment }\end{array}$ & AND, OR & 10 \\
PsycINFO & $\begin{array}{l}\text { Mindfulness, TDAH, ADHD, terapia, therapy, } \\
\text { tratamiento, treatment }\end{array}$ & AND, OR & 2 \\
RODERIC & $\begin{array}{l}\text { Mindfulness, TDAH, ADHD, terapia, therapy, } \\
\text { tratamiento, treatment }\end{array}$ & AND, OR & 0 \\
Google scholar & $\begin{array}{l}\text { Mindfulness, TDAH, ADHD, terapia, therapy, } \\
\text { tratamiento, treatment }\end{array}$ & AND, OR & 41 \\
PubMed & $\begin{array}{l}\text { Mindfulness, TDAH, ADHD, terapia, therapy, } \\
\text { tratamiento, treatment }\end{array}$ & AND, OR & 60 \\
\hline
\end{tabular}

Total

Tabla 1. Resultados de la primera fase de la revisión bibliográfica Fuente: Elaborado por las autoras. 
En la segunda fase de la revisión se concretaron unos primeros criterios de inclusión y exclusión. Se establecieron así una serie de parámetros que delimitaron los resultados de la búsqueda de documentos para que ésta resultase más efectiva y respondiera de manera adecuada a las necesidades del objeto de estudio. Los criterios de inclusión utilizados fueron los siguientes: 1. Se establece un período de publicación de los documentos buscados y elegidos desde 2001 hasta la actualidad 2018, porque nos hemos centrado en documentos más recientes y actuales, intentando evitar información fuerza obsoleta. 2. El idioma elegido para la documentación ha sido tanto el español como el inglés para ampliar la búsqueda, dada la existencia de un volumen importante de bibliografía sobre el tema en lengua inglesa. 3. Los estudios incluirán una muestra diagnosticada con TDAH. 4. Los estudios deben abordar una población de niños/as $\mathrm{y}$ adolescentes.

Los criterios de exclusión fueron los siguientes: 1. Estudios científicos publicados en forma de resumen, es decir, aquellos que no proporcionaban el texto completo, ya que de esta manera no aportaban suficiente información para la revisión. 2. Trabajos en idioma diferente al español e inglés. 3. Estudios en los que la muestra no ha sido diagnosticada con TDAH. 4. Estudios en que la intervención no tiene como elemento central el mindfulness. 5. Estudios en que la muestra no son niños/as y/o adolescentes, para contextualizarla en la etapa de la infancia o la adolescencia.

En la primera fase del estudio se obtuvieron como resultado 121 referencias. Con la utilización de los criterios se excluyen 109 artículos y se procede a elegir los artículos a texto completo para su análisis seleccionando 12 artículos. Finalmente, se incluyen en el estudio 6 artículos que cumplían estrictamente con los criterios de selección establecidos.

En la tercera fase de la revisión bibliográfica se elaboraron los resultados clasificando los artículos analizados en dos apartados que se relacionaban con la finalidad y objetivos de la investigación: Revisiones bibliográficas sobre la efectividad del mindfulness en el contexto educativo y revisiones bibliográficas sobre las intervenciones basadas en mindfulness realizadas con niños/as con TDAH.

\section{RESUltados}

\subsection{REVISIONES BIBLIOGRÁficA SOBRE LA EFECTIVIDAD DEL MINDFULNESS EN EL CONTEXTO EDUCATIVO}

En la tabla 2 se muestran los resultados más relevantes obtenidos en la revisión bibliográfica realizada sobre la efectividad del mindfulness en el contexto educativo. 


\begin{tabular}{|c|c|c|}
\hline Autor & Noestudios & Resultados \\
\hline $\begin{array}{l}\text { Meiklejohn et al. } \\
\text { (2012) }\end{array}$ & $\mathrm{N}=14$ estudios & Beneficios cognitivos, sociales y psicológicos \\
\hline $\begin{array}{l}\text { Zenner et al. } \\
(2014)\end{array}$ & $\mathrm{N}=24$ estudios & $\begin{array}{l}\text { Mejora en el dominio del ejercicio cognitivo, y resultados significati- } \\
\text { vos en medidas de estrés, afrontamiento y resiliencia. }\end{array}$ \\
\hline $\begin{array}{l}\text { Zoogman et al., } \\
\text { (2015) }\end{array}$ & $\mathrm{N}=20$ estudios & $\begin{array}{l}\text { Mejor efectividad en síntomas psicológicos, lo que comporta el bene- } \\
\text { ficio para poblaciones clínicas. }\end{array}$ \\
\hline $\begin{array}{l}\text { Waters et al., } \\
(2015)\end{array}$ & $\mathrm{N}=15$ estudios & $\begin{array}{l}\text { Pequeño efecto significativo sobre el bienestar y la competencia social } \\
\text { de los alumnos. }\end{array}$ \\
\hline Felver et al. (2016) & $\mathrm{N}=28$ estudios & $\begin{array}{l}\text { Mejora de resultados educativos y psicosociales de nińos/as y adoles- } \\
\text { centes. }\end{array}$ \\
\hline $\begin{array}{l}\text { Klingbeil et al., } \\
(2017)\end{array}$ & $\mathrm{N}=76$ estudios & $\begin{array}{l}\text { Pequeños efectos en logros académicos, problemas externalizantes e } \\
\text { internalizantes, emociones negativas y angustia, emociones positivas y } \\
\text { valoración de uno mismo, salud física y finalmente competencia social } \\
\text { y comportamientos pro-sociales. }\end{array}$ \\
\hline
\end{tabular}

Tabla 2. Información evidencia científica de la eficacia del mindfulness en el alumnado respecto a revisiones y meta-análisis realizados

Fuente: Elaborado por las autoras.

\subsection{REVISIÓN BIBLIOGRÁFICA SOBRE LAS INTERVENCIONES BASADAS EN MINDFULNESS REALIZADAS CON NIŃOS/AS Y ADOLESCENTES CON TDAH}

En la tabla 3 se muestra la información más relevante de los seis artículos seleccionados sobre las intervenciones basadas en mindfulness realizadas con nińos/as y adolescentes con TDAH: autores del estudio y año de publicación, tamaño de la muestra, edad y diagnóstico, así como la existencia de grupo control o no al estudio.

\begin{tabular}{|c|c|c|c|c|c|}
\hline Autor & $\begin{array}{l}\text { Características } \\
\text { muestra }\end{array}$ & Evaluación & Programa & Diseño & Resultados \\
\hline $\begin{array}{l}\text { Meppelink, } \\
\text { Bruin, y Bögels } \\
\text { (2016) }\end{array}$ & $\begin{array}{l}\mathrm{N}=120 \text { familias con } \\
\text { niños/as con TDAH } \\
\text { entre } 9 \text { y } 18 \text { ańos }\end{array}$ & $\begin{array}{l}\text { DBDRS } \\
\text { CBCL } \\
\text { TRF } \\
\text { YSR } \\
\text { TRF } \\
\text { TEA-Ch }\end{array}$ & $\begin{array}{l}\text { Protocolo } \\
\text { Mymind }\end{array}$ & $\begin{array}{l}\text { Estudio aleatorio }(\mathrm{N}=60) \\
\text { grupo mindfulness, }(\mathrm{N}=60) \\
\text { medicación } \\
\text { seguimiento ( } 4 \text {-10 meses). } \\
\text { No grupo control. } \\
\text { Pre-test-Post-test } \\
\text { Intervención paralela a } \\
\text { padres y hijos. }\end{array}$ & $\begin{array}{l}\text { La intervención mindful- } \\
\text { ness puede ser una alterna- } \\
\text { tiva a la medicación. }\end{array}$ \\
\hline
\end{tabular}




\begin{tabular}{|c|c|c|c|c|}
\hline \multirow{5}{*}{$\begin{array}{l}\text { Lo, Wong, } \\
\text { Wong, Wong, y } \\
\text { Yeung (2016) }\end{array}$} & & ANT & \multirow{5}{*}{$\begin{array}{l}\text { Protocolo } \\
\text { FBMI }\end{array}$} & \multirow{3}{*}{$\begin{array}{l}\text { Estudio aleatorio con } \\
\text { grupo control. }\end{array}$} \\
\hline & $\mathrm{N}=120$ (niños/as entre & $\begin{array}{l}\text { PSI-SF } \\
\text { HRV }\end{array}$ & & \\
\hline & 5 y 7 ańos y sus padres) & CBCL & & \\
\hline & Diagnóstico TDAH & ASRS & & Seguimiento 8 y 10 \\
\hline & 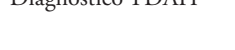 & $\begin{array}{l}\text { IMP } \\
\text { SWAN-C }\end{array}$ & & semanas. \\
\hline
\end{tabular}

Van de
Weijer-Bergsma,

Formsma, De

Bruin, y Bögels

(2012)

$\begin{array}{ll}\begin{array}{ll}\text { N=10 (5 chicas i } 5 \\ \text { chicos entre } 11 \text { y } 15\end{array} & \text { BRIEF; } \\ \text { ańos }) & \text { CBCL; } \\ & \text { MAAS; PSI; } \\ \text { Diagnóstico TDAH } & \begin{array}{l}\text { YSRF; } \\ \text { SHS; FSS; }\end{array} \\ 19 \text { padres y } 7 \text { tutores. } & \text { ANT }\end{array}$

$\mathrm{N}=22$ (16 niños y 6

Bögels,y Peijnenburg (2012) niñas entre 8 y 12 ańos)

Diagnóstico TDAH

21 madres y 1 padre

DBDRS

PSI

PS

MAAS

ARS

$\mathrm{N}=4$ (2 niños entre $10 \mathrm{i}$

12 ańos y sus madres)

$$
\text { Diagnóstico TDAH }
$$

SSIMC
Singh et al. (2009)
Pre-test 1 semana antes, Post-test después de las 8 semanas de tratamiento.

No grupo control

Seguimiento a las 8 y 16 semanas

después del post-test.

Pre-test 1 semana antes, Post-test al acabar la intervención.

Grupo de control

Seguimiento a las 8

semanas de finalizar el

tratamiento.

Cuestionario profesores.

Diseńo de referencia

Protocolo de

12 semanas

SUHMC múltiple.

No grupo control

Entrenamiento de 12

semanas de duración,

seguida de 12 semanas de

entrenamiento a los nińos.

Seguimiento 24 semanas.
Reducción en nińos/as de los síntomas de hiperactividad y de inatención en comparación con el grupo control.

Los padres manifiestan menor estrés y mayores habilidades parentales.

Reducción de los problemas de atención (padres). Reducción en la internalización y externalización de problemas (padres y adolescentes) Madres y tutores no coinciden en los resultados comentados Mejora en las funciones ejecutivas (padres y tutores)

Reducción en la sintomatología TDAH (padres e hijos).

Reducción del estrés parental.

Los datos de los profesores no son significativos respecto a la reducción de síntomas.

Incremento en el cumplimiento de las peticiones de las madres por parte de los niños.

Incremento en la

satisfacción en la crianza y interacción con sus hijos.

Transformaciones

personales en madres $y$ hijos en lugar de enseńar estrategias para el cambio de conducta.

Tabla 3. Información relevante sobre el diseño y los resultados de las investigaciones seleccionadas

Fuente: Elaborado por las autoras.

Measures: ANT: Attitudes towards Neuroleptic Treatment; ARS: ADHD Rating Scale; ASRS: Adult ADHD Self Report Scale; BAI: Beck Anxiety Inventory; BDI: Beck Depression Inventory; BRIEF: Behaviour Rating Inventory of Executive Function; CAARS: Conners' Adult ADHD Rating Scales; CBCL: Child Behaviour Checklist; DBDRS: Disruptive Behaviour Disorders Rating Scale;FFS: Flinders Fatigue Scale; HRV Parent heart rate variability; IM-P: Interpersonal Mindfulness in Parenting; MAAS: Mindful Attention Awareness Scale; PS: Parenting Scale; PSI: Parenting Stress Index; SHS: Student Health Services; SSIMC: Satisfaction with self in interactions

En este caso destacamos la escasa utilización de grupos control. Como podemos ver en la figura 2, solo el 33\% lo utiliza, frente al $67 \%$ que no utiliza grupo control. Asimismo, se incluyen los instrumentos empleados para la evaluación, tipo y diseño de la intervención y los resultados obtenidos en el estudio. 
Todos los estudios se han realizado con niños/as y adolescentes diagnosticados con TDAH y sus padres. Tres estudios se realizan con una muestra reducida (Singh et al., 2009; Van de Weijin-Bergsma et al., 2012), cinco estudios realizan con los niños/as y adolescentes un entrenamiento simultáneo con los padres (Lo et al., 2016; Meppelink et al., 2016; Van de Oord et al., 2012; Van de Weijin-Bergsma et al., 2012) y en uno se realiza un entrenamiento de 12 semanas con mindfulness con las madres y luego 12 semanas de entrenamiento con el niños (Singh, et al., 2009).

En general las intervenciones tienen un efecto beneficioso para toda la familia. En concreto tres estudios muestran una reducción en los niveles de estrés parental (Lo et al. 2016; Van de Oord et al., 2012) y dos muestran una mejora en las habilidades parentales (Lo et al., 2016; Singh et al., 2009) y en uno se muestra un incremento en la satisfacción respecto a la crianza e interacción con los hijos (Singh, et al. 2009).

Como vemos en la Figura 3, en el $67 \%$ de las intervenciones se utiliza el programa "MyMind" programa de intervención para padres e hijos con TDAH, desarrollado y validado en 2008 por Susan Bögels. El 37\% restante utiliza otros tipos de programas basados en mindfulness con el FMBI de ocho semanas (Lo et al., 2016) y un protocolo de 12 semanas de entrenamiento en mindfulness (Singh, et al., 2009).

Respecto a la duración de las intervenciones del presente estudio cinco de ellas tienen una duración de ocho semanas (Lo et al., 2016; Meppelink et al., 2016; Van de Oord et al., 2012; Van de Weijin-Bergsma et al., 2012). En uno de los estudios la duración de la intervención sería de 12 semanas con las madres y después 12 semanas con los niños/as y adolescentes (Singh, et al., 2009). También en todas las intervenciones se establece un periodo de seguimiento que iría entre las ocho y las 24 semanas a la finalización del tratamiento.

Como vemos en la figura 4, en referencia a los resultados obtenidos en relación con la sintomatología característica del TDAH, el $40 \%$ de los estudios muestra una mejoría respecto a la inatención, un 30\% muestra una mejoría respecto a la hiperactividad y otro $30 \%$ muestra una mejoría respecto a la impulsividad. También en uno de los estudios se muestra un incremento en el desempeño de las peticiones de las madres por parte de los niños/as (Singh et al., 2016).

\section{Conclusiones}

En la primera fase de la revisión realizada pudimos concluir que existe un número importante de artículos y estudios referentes a las intervenciones de TDHA basadas en mindfulness. De la revisión bibliográfica inicial, finalmente sólo seis artículos cumplirían los criterios establecidos para concretar la búsqueda en intervenciones que tienen como elemento principal el mindfulness y que han sido aplicadas en niños/as diagnosticados/as con TDAH. El cumplimiento de estos parámetros nos permite contextualizar estas intervenciones en el alumnado que presenta este trastorno y ubicarlas en la franja educativa de la infancia y la adolescencia. Así pues, en función de estos resultados se plantea la necesidad de realizar más estudios sobre los beneficios y la efectividad que este tipo de intervenciones pueden tener para el alumnado con TDAH, teniendo en cuenta el diagnóstico, la edad y el contexto en el que se lleva a cabo la intervención. Aunque los resultados mostrados por los estudios son esperanzadores, es importante poner de relieve que ninguna de estas intervenciones son llevadas a cabo en el con- 
texto educativo, aspecto fundamental para la realización de una intervención inclusiva de este alumnado. Para fomentar la inclusión se deberían aplicar programas educativos inclusivos de mindfulness donde se tengan en cuenta las necesidades de todo el alumnado, independientemente si tiene TDAH o no. En este caso, se abre la necesidad de iniciar futuras investigaciones que vayan en línea a dar respuesta a este aspecto.

La revisión realizada también permite concluir la existencia de un importante volumen de estudios sobre los beneficios cognitivos, psicológicos y sociales que el mindfulness puede aportar en su aplicación al contexto educativo. La revisión realizada sobre la eficacia del mindfulness en población clínica con TDAH nos permite concluir la necesidad de realizar estudios y experiencias prácticas que muestren los beneficios psicosociales que a su vez pueden aportar estas intervenciones en este alumnado, no sólo en el contexto familiar, sino al contexto educativo, tal y como muestran el resto de los estudios, revisiones y meta-análisis mencionados. Para ello, es necesario fomentar programas de formación para el profesorado para que tengan estrategias y confianza para aplicarlo en sus aulas.

Los artículos analizados también muestran una serie de intervenciones realizadas en población clínica diagnosticada con TDAH y llevadas a cabo en el contexto familiar principalmente, lo que reafirma la falta de evidencia empírica generalizada sobre la efectividad que estas intervenciones pueden tener en otros contextos como el educativo. Además, podemos concluir la existencia de diferentes limitaciones metodológicas generales como la medida de la muestra, ya que tres de los estudios de la revisión presentan una muestra reducida; la falta de estudios cualitativos, ya que del resultado inicial de la investigación se seleccionan solamente seis estudios que cumplen con los diferentes criterios establecidos; falta de estandarización en las medidas de evaluación porque como vemos no hay uniformidad en los instrumentos de evaluación utilizados en los diferentes estudios; y, por último, la falta de grupos control en los estudios de las intervenciones basadas en mindfulness.

En conclusión, a pesar de la efectividad mostrada en la revisión de los tratamientos basados en mindfulness sobre las sintomatologías características del TDAH y para futuras líneas de investigación se hace necesaria la realización de más investigaciones con un mayor rigor metodológico, que presenten un tamaño de la muestra adecuada, con un diagnóstico del trastorno bien establecido, edad de la muestra en la franja de la infancia y la adolescencia, grupo control, unas medidas de evaluación estándar y que además su aplicación no se limite al contexto familiar y clínico, es decir, que se contemplen otros contextos como el educativo. La posibilidad de ofrecer a este alumnado un tratamiento basado en mindfulness que se lleve a cabo junto al contexto familiar, el contexto educativo y de manera inclusiva podría ayudar a todo el alumnado, no solamente a los alumnos de TDAH, a reducir el estrés y aumentar su propio bienestar, para alcanzar y optimizar el aprendizaje y mejorar sus relaciones interpersonales, enriqueciendo así su forma de estar y actuar en el mundo (Zenner et al., 2014). 


\section{REFERENCIAS}

American Psychiatric Association (2014). DSM-5: Manual diagnóstico y estadístico de los trastornos mentales (5a ed.). Madrid: Editorial Médica Panamericana.

Anand, U., y Sharma, M. P. (2014). Effectiveness of a mindfulness-based stress reduction program on stress and well-being in adolescents in a school setting. Indian Journal of Positive Psychology, 5(1), 17.

Barkley, R., y Murphy, K. (2010). Deficient emotional self-regulation in adults with Attention-Deficit/ Hyperactivity Disorder (ADHD): The relative contributions of emotional impulsiveness and ADHD symptoms to adaptive impairments in major life activities. Journal of ADHD \& Related Disorders, 1(4), 5-28.

Biegel, G., y Brown, K. W. (2010). Assessing the efficacy of an adapted inclass mindfulness-based training program for school-age children: A pilot study. Mindful Schools. Recuperado de https:// goo.gl/3rnRd1.

Bögels, S., Hoogstad, B., Van Dun, L., De Shutter, S., y Restifo, K. (2008). Mindfulness training on the behavior of elementary students with attention-deficit/hiperactive disorder. Research in Human Development, 10(3), 234-251.

Britton, W. B., Lepp, N. E., Niles, H. F., Rocha, T., Fisher, N. E., y Gold, J. S. (2014). A randomized controlled pilot trial or clasroom base mindfulness meditation compared to an active control condition in sixth-grade children. Journal of School Psychology, 52(3), 263-278.

Broderick, P. C., y Metz, S. (2009). Learning to Breathe: A pilot trial of mindfulness currículum for adolescents. Advances in School Mental Health Promotion, 2(1), 35-46.

Catalá-López, F., y Hutton, B. (2018). The treatment of attention deficit hyperactivity disorder in children and adolescents: Epidemiology, multimorbidity and integrated health services. Anales de pediatría, 88(4), 181-182.

Desmond, C. T., Hanich, L., y Millersville, P. A. (2010). The effects of mindful awarenes teaching practices on the executive funcions of students in an urban, low income middle school. Millervilles: Millerville University.

Felver, J. C., Celis-de Hoyos, C. E., Tezanos, K., y Singh, N. N. (2016). A systematic review of mindfulness-based interventions for youth in school settings. Mindfulness, 7(1), 34-45.

Flook, L., Smalley, S. L., Kitil, M. J., Galla, B. M., Kaiser-Greenland, S., Locke, J. ... Kasari, C. (2010). Effects of mindful awareness practices on executive funcions in elementary school children. Journal of Applied School Psychology, 26(1), 70-95.

Franco, C. (2009). Efectos de un programa de meditación sobre los niveles de creatividad verbal de un grupo de alumnos/as de Bachillerato. Suma Psicológica, 16(2), 113-120.

Franco, C., Mañas, I., Cangas, A. J., y Gallego, J. (2011). Exploring the effects of a mindfulness proram for students of secondary school. International Journal of Knowledge Society Research (IJKSR), 2(1), 14-28.

García, J., Demarzo, M., y Modregó, M. (2017). Bienestar emocional y midfulness en la educación. Madrid: Alianza editorial.

Joyce, A., Etty-Leal, J., Zazryn, T., y Hamilton, A. (2010). Exploring a mindfulness meditation program on the mental health of upper primary children: A pilot study. Advances in School Mental Health Promotion, 3(2), 17-18.

Kabat-Zinn, J. (2003). Mindfulness based intervention in context: Past, present and future. Clinical Psychology: Science and Practice, 10(2), 144-146. 
Klatt, M., Harpster, K., Browne, E., White, S., y Case-Smith, J. (2013). Feasibility and preliminary outcomes for move-into-learning. An arts based mindfulness classroom intervention. The journal of Positive Psychology, 8, 233-241.

Klingbeil, D. A., Renshaw, T. L., Willenbrink, J. B., Copek, R. A., Chan, K.T., Haddock, A., y Clifton, J. (2017). Mindfulness-based interventions with youth: A comprehensive meta-analysis of group design studies. Journal of School Psychology, 63, 77-103.

Kuyken, W., Weare, K., Ukoumunne, C., Vicary, R., Motton, N., Burnett, R. ... Huppert, F. (2013). Effectiveness of the Mindfulness in Schools Programme: Non-randomised controlled feasibility study. The British Journal of Psychiatry. 203(2), 126-131.

León, B. (2008). Atención plena y rendimiento académico en estudiantes de educación secundaria. European Journal of Education and Psychology, 1(3), 17-26.

Lo, H., Wong, S., Wong, J., Wong, S., y Yeung, J. (2016). The effect of a family-based mindfulness intervention on children with attention deficit and hyperactivity symptoms and their parents: Design and rationale for a randomized, controlled cliniccal trial (study control). BMC Psychiatry, 16, 65. Recuperado de https://goo.gl/DkfVVf.

Meiklejohn, J., Phillips, C., Freedman, M. L, Griffin, M. L., Biegel, G., Roach, A. ... Saltzman, A. (2012). Integrating mindulness training into K-12 education: Fostering the resilience of teachers and students. Mindfulness, 3(4), 291-307.

Meppelink, R., Bruin, E., y Bögels, S. (2016). Meditation or medication? Mindfulness training versus medication in the treatment of childhood ADHD: A randomized controlled trial. BMC Psychiatry, 16, 267.

Metz, S. M., Frank, J. L., Reibel, D., Cantrell, T., Sanders, R., y Broderick, P. C. (2013). The effectiveness of the learning to Breathe program on adolescent emotion regulation. Research in Human Development, 10(3), 252-272.

Miranda, A., Jarque, S., y Tárraga, R. (2006). Interventions in school settings for students with ADHD. Exceptionality, 14, 35-52.

Napoli, M., Krech, P. R., y Holley, L.C. (2005). Mindfulness training for elementary school students: The attention academy. Journal of Applied School Psychology, 21(1), 99-125.

Orjales, I. (2000). Déficit de atención con hiperactividad: El modelo híbrido de las funciones ejecutivas de Barkley. Revista Complutense de Educación, 11(1), 71-84.

Potek, R. (2012). Minduflness as a school. based prevention program and its effect on adolescent stress, anxiety and emotion regulation, New York: New york University.

Razza, R., Martin, A., y Brooks-Gunn, J. (2012). The Implications of Early Attentional Regulation for School Success among Low-Income Children. Journal of Applied Developmental Psychology, 33(6), 311-319.

Rechtscgaffen, D. J. (2016). Educación mindfulness. El cultivo de la consciencia y la atención para profesores y alumnos. Madrid: Gaia Ediciones.

Rosaen, C., y Benn, R. (2006). The experience of transcendental meditation in middle school students: A qualitative report. Explore: The Journal of Science and Healing, 2, 422-425.

Saltzman, A., y Goldin, P. (2008). Mindfulness based stress reduction for school-age children. En S. C. Hayes, y L. A. Greco (Eds), Acceptance and mindfulness interventions for childresn adolescents and families (pp.139-161). California: New Harbinger. 
Sánchez, N., y González, C. (2013). Ajuste escolar del alumnado con TDAH: Factores de Riesgo Cognitivos, Emocionales y Temperamentales. Electronic Journal of Research in Educational Psychology, 11(2), 527-550.

Schachter, H. M., King, J., Langford, S., y Moher, D. (2001). How efficacious and safe in short-acting methylphenidate for the treatment of attention-deficit disorder inchildren and adolescents? A meta-analysis. Canadian Medical Association Journal, 165(11), 1475-1488.

Schonert-Reichl, E., y Lawlor, M. S. (2010). The effects of a mindfulness-based educaion progam on preand early adolescents' well-being and social and emotional competence. Mindfulness, 1(3), 137-151.

Schonert-Reichl, E., Oberle, E., Lawlor, M. S., Abbott, D., Thomson, K., Oberlander, T. F., y Diamond, A. (2015). Enhancing cognitive and social-emotional development through a simple-to-administer mindfulness-based school program for elementary school children: A randomized controlled trial. Developmental. Psychology, 51, 52-56.

Singh, N. N., Singh, A. N., Lancioni, G. E., Singh, J., Winton, A. S., y Adkins, A. D. (2009). Mindfulness training for parents and their children with ADHD increases the children's compliance. Journal of Child and Family Studies, 19, 157-166.

So, K. T., y Orme-Johnson, D. W. (2001). Three randomized experiments on the longitudinal effects of the Transcedental Meditation technique on cognition, Intelligence, 29(5), 419-440.

Tang, Y. Y., Yang, L., Leve, L. D., y Harold, G. T. (2012). Improving executive function andits neurobiological mechanisms through a mindfulness-based intervention: Advances within the field of developmental neuroscience. Child Development Perspectives, 6, 361-366.

Van de Oord, S., Bögels, S., y Peijnenburg, D. (2012). The effectiveness of mindfulness training for children with ADHD and mindful parenting for their parents, Journal of Child and Family Studies, 21, 139-147.

Van de Weijer-Bergsma, E., Formsma, A. R., De Bruin, E. I., y Bögels, S. M. (2012). The effectiveness of mindfulness training on behavioral problems and attentional functioning in adolescents with ADHD. Journal of Child and Family Studies, 21(5), 775-787.

Wall, R. B. (2005). Tai chi and mindfulness-based stress reduction in a Boston public middle school. Journal of Pediatric Health Care, 19(4), 230-237.

Waters, L., Barsky, A., Ridd, A., y Allen, K. (2015). Contemplative education. A systematic, evidencebased review of the effect of mediation interventions in schools. Educational Psychology Review, 27(1), 103-134.

Zenner, C., Herrleben-Kurz, S., y Walach, H. (2014). Mindfulness-based interventions in schools: A systematic review and meta-analysis. Frontiers in Psychology, 5, 603. Recuperado de https://goo.gl/b91Buu.

Zoogman, S., Goldberg, S. B., Hoyt, W. T., y Miller, L. (2015). Mindfulness interventions with youth: A meta-analysis. Mindfulness, 6(2), 290-302. ${ }^{4}$

Recibido en:14/07/2018

Reformulado en: 27/10/2018

Aprobado en: 29/10/2018 